\title{
Sats in the 60s
}

\author{
Raquel M. Schears • Jamie M. Hess
}

Received: 10 January 2008 / Accepted: 29 January 2008 /Published online: 15 March 2008

(C) Springer-Verlag London Ltd 2008

A 61-year-old man presented to the emergency department (ED) with sudden onset shortness of breath, coughing paroxysms, and a brief loss of consciousness. The emergency medical services (EMS) noted the patient to be diaphoretic and unresponsive, with a prior history of single lung transplantation. His current medications were multiple, without known allergy. Upon ED arrival, the patient was alert and oriented with normal vital signs, except for a marked tachypnea and saturations of $64 \%$ on $100 \%$ oxygen via a non-rebreather face mask. The initial chest radiograph shows a density in the left upper lung field, missed on the radiologist's reading of the film (Fig. 1a, arrow). Shortly thereafter, the patient began to cough violently and expelled a large pellet of chalk-like material, ascertained to be the remnants of a calcium supplement. Post expulsion, the oxygen saturation immediately rose to $99 \%$ and his respiratory rate decreased to 14 . A repeat chest radiograph revealed an absence of the calcified density in the left upper lobe, and clear reduction of adjacent parenchymal atelectasis (Fig. 1b). Clinicians should consider the possibility of pill aspiration on the differential for sudden marked hypoxia and tachypnea, especially in post lung transplant patients.

R. M. Schears $(\square)$

Department of Emergency Medicine,

Mayo Clinic College of Medicine, Mayo Clinic,

200 First Street SW,

Rochester, MN 55905, USA

e-mail: schears.rocky@mayo.edu

\section{J. M. Hess}

Mayo Clinic College of Medicine, Mayo Clinic,

Rochester, MN, USA

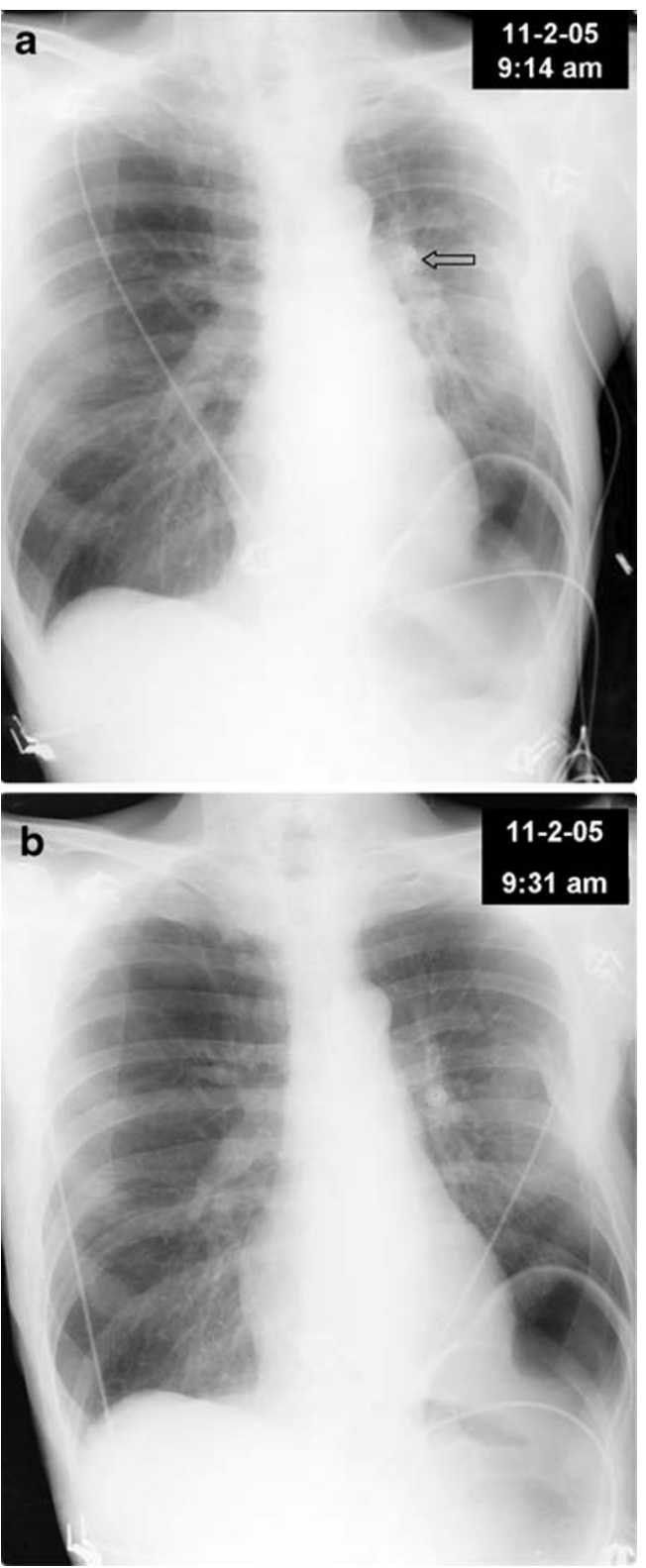

Fig. 1 a Initial and $\mathbf{b}$ repeat chest radiographs 